\title{
Nanoscale Magnetic Characterization of TMR Spin Valve by Electron Holography
}

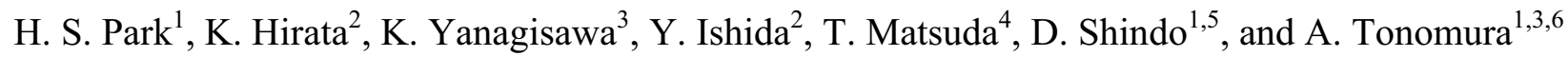 \\ 1. Advanced Science Institute, RIKEN, Wako, Saitama 351-0198, Japan. \\ 2. Head Business Group, TDK Corporation, Saku, Nagano 385-8555, Japan. \\ 3. Okinawa Institute of Science and Technology Graduate University, Okinawa 904-0495, Japan. \\ 4. Japan Science and Technology Agency, Kawaguchi-shi, Saitama 332-0012, Japan. \\ 5. IMRAM, Tohoku University, Sendai, Miyagi 980-8577, Japan. \\ ${ }^{6 .}$ Central Research Laboratory, Hitachi, Ltd., Hatoyama, Saitama 350-0395, Japan.
}

With the discovery of giant magnetoresistance (GMR) [1], read heads and magnetic sensors for hard disk drives have been developed, not only in design but also in more complicated fabrication, that are scaled down to nanometers (nm). This has led to an increase in areal density recording by three orders of magnitude (from 0.1 to $100 \mathrm{Gbit} / \mathrm{in}^{2}$ ) and the emergence of spin-electronic devices such as magnetic random access memory. However, there are many factors that are still unclear and that have a negative effect on the MR effect and spin electronics, i.e., interfacial and barrier spin scattering, magnetic flux leakage, and changes in the output voltage. Also problematic is the increasing miniaturization from micrometers to $\mathrm{nm}$ in devices. For investigation of these problems, mainly associated with spin, nanoscale imaging of magnetic field is indispensable. However, the magnetization distribution in real device structures has not yet been investigated on the $\mathrm{nm}$ scale due to device complexity and/or the insufficient spatial resolution of the microscopes available. This motivated us to perform electron holography observations of such real device structures with high spatial and phase resolutions.

The specimen studied was selected from a tunneling magnetoresistance (TMR) head in production. A thin film specimen was fabricated using the focused ion beam (FIB) method combined with microsampling. The primary reason for using FIB was to prepare a thin film with a uniform thickness below $100 \mathrm{~nm}$ without damaging a layer during milling. However, the thin film turned out to have a wedge shape due to milling at a low acceleration voltage of $5 \mathrm{kV}$ to minimize layer damage. The thin film was placed in a special specimen holder designed for both observing and FIB milling, which enabled us to flip the specimen $180^{\circ}$. A holography electron microscope (HF-3300X, Hitachi) was utilized for all experiments. This microscope has two positions in which the specimen can be placed, i.e., the field-free position and the objective lens position. Electron holograms (under no applied field) were observed in the field-free position where the residual magnetic field was approximately $0.01 \mathrm{mT}$.

Figure 1 depicts the schematic illustration of TMR spin valve head and its reconstructed phase images representing electric potential $\left(\varphi_{\mathrm{E}}\right)$ and magnetic vector potential $\left(\varphi_{\mathrm{M}}\right)$, in which the phase shift $\varphi(x, y)$ is represented by $\cos \varphi(x, y)$. The complex hetero-nanostructures of the spin valve head were observed, which consist of various materials with magnetic or nonmagnetic properties. Depending on their functional properties, they are designated as cap $\left(\mathrm{C}_{1}, \mathrm{C}_{2}\right)$, insulator (I), upper shield (US), lower shield (LS), bias (B), free $(\mathrm{F})$, and pinned $(\mathrm{P})$ layers. In order to separate the phase shifts affected by superimposed electric and magnetic vector potentials, we flipped the specimen from $0^{\circ}$ to $180^{\circ}$ and recorded two holograms where only the sign of the phase shift due to the magnetic vector potential was reversed. By simple summation and subtraction of those two phase images [2], $\varphi_{\mathrm{E}}$ and $\varphi_{\mathrm{M}}$ can be separated, as shown in Figure 1. Given measured thicknesses, the magnetic flux densities of the US, B, and $\mathrm{F}$ layers were estimated to be $1.0 \mathrm{~T}, 0.9 \mathrm{~T}$, and $1.0 \mathrm{~T}$, respectively. 
Directly visualized in the right panel of Figure 1 is the magnetization distribution by separating the electric potential and averaging phase images reconstructed from ten holograms consecutively taken under the same conditions, which provides improved phase resolution. In the magnetic flux map, the direction and the density of black (or white) contour lines correspond to those of the in-plane component of the magnetic flux density projected along the electron beam. The thick contour lines were clearly observed in both the $\mathrm{NiFe}$ (not colored) and CoPt layers (colored in blue). Furthermore the contour line was visible inside the $5 \mathrm{~nm} \mathrm{NiFe}$ (free layer) below the dotted line in yellow colored region. In the CoFe layer (colored in red), the in-plane component of the magnetic flux seems to be zero (or extremely small), meaning that its magnetization direction is normal to the film plane. The change in the magnetization distribution with an applied field of $14 \mathrm{kOe}$ normal to the film plane, like that of actual device operation, will be also presented. The methodology reported here [3], electron holography, is very promising for visualizing and quantitatively characterizing magnetic properties in devices on the $\mathrm{nm}$ scale, which may improve TMR head design and solve problems caused by complexity and miniaturization in spin-electronic devices.

\section{References:}

[1] A. Fert, P. Grunberg, K. Grandin, ed. Les Prix Nobel, The Nobel prizes 2007, (Stockholm, 2008).

[2] A. Tonomura, Electron holography, 2nd ed., Springer-Verlag, Tokyo, 1999.

[3] H. S. Park et al, Small, 8 (2012), p. 3640.

[4] This research was supported by the grant from the Japan Society for the Promotion of Science (JSPS) through the "Funding Program for World-Leading Innovative R\&D on Science and Technology (FIRST Program)," initiated by the Council for Science and Technology Policy (CSTP).
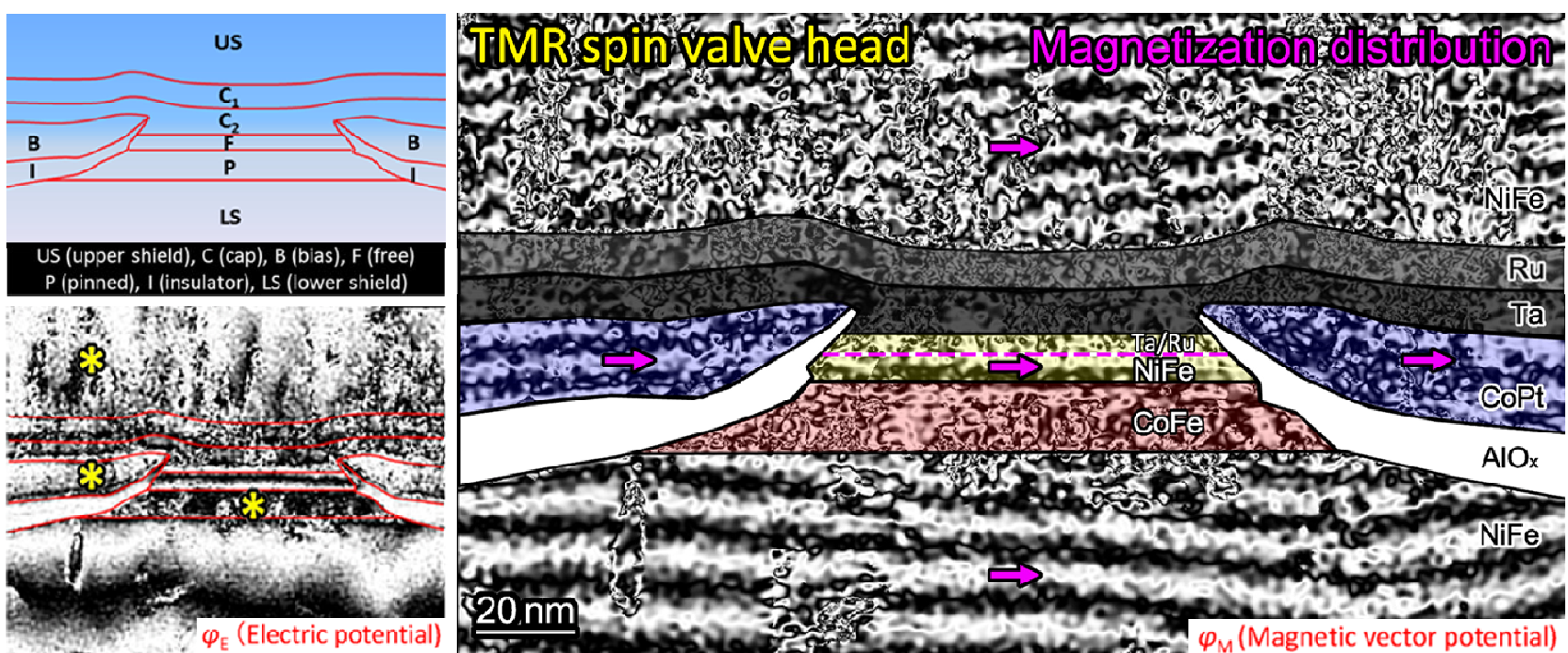

Figure 1. TMR spin valve head structures. Equipotential contour map (left lower panel, amplified by 2) represents the projected thickness and the mean inner potential of materials whereas magnetization distribution (right panel, amplified by 6) reveals the in-plane component of the magnetic flux in this viewing field. Asterisks and arrows indicate the positions for thickness measurement and the magnetization direction, respectively. (For interpretation of the references to color in this figure legend, the reader is referred to the web version of this article.) 